\title{
Association of infant diet with subsequent obesity at 2-5 years among children exposed to gestational diabetes: the SWIFT study
}

\author{
Sarvenaz Vandyousefi ${ }^{1}$ (D) Jaimie N. Davis ${ }^{2}$ (D) Erica P. Gunderson ${ }^{3}$ (D) \\ Received: 15 September 2020 / Accepted: 2 November 2020 / Published online: 26 January 2021 \\ (C) The Author(s), under exclusive licence to Springer-Verlag GmbH, DE part of Springer Nature 2021
}

\begin{abstract}
Aims/hypotheses This longitudinal analysis evaluated the independent and joint associations of any breastfeeding (BF) or exclusive BF (EBF) and intake of sugar-sweetened beverages (SSBs) and 100\% fruit juice from birth to 1 year with subsequent overweight and obesity among young children exposed to gestational diabetes (GDM).

Methods The analysis utilised prospectively collected data from participants enrolled in the Study of Women, Infant Feeding and Type 2 Diabetes after GDM (SWIFT); 1035 pregnant women (20-45 years) diagnosed with GDM, of whom 75\% were of Black, Hispanic or Asian race and ethnicity. Mother-infant dyad characteristics and infant dietary intake were assessed via research protocols at in-person examinations, telephone interviews and monthly mailed surveys from birth to 1 year. Child weight, length and height were obtained from electronic health records at birth (2008-2011) and ages 2-5 years (2010-2016) to classify BMI percentile groups $(n=835)$.

Results Adequate BF ( $\geq 6$ months), adequate EBF duration ( $\geq 6$ months), and SSB and $100 \%$ fruit juice intake in the first year were independently associated with child obesity at ages $2-5$ years (all $p<0.05$ ). Compared with children with adequate EBF and no intake of SSB or $100 \%$ fruit juice, those with adequate EBF and intake of $100 \%$ fruit juice and/or SSBs had a four- to fivefold higher odds of obesity (aOR 4.2, 95\% CI:1.6, 11.2 for 100\% fruit juice; aOR 4.5, 95\% CI:1.4, 8.5 for fruit juice or SSBs; and aOR 4.7, 95\% CI:1.4, 15 for SSBs; all $p<0.01$ ), while those with inadequate EBF ( $<6$ months) and intake of $100 \%$ fruit juice and/or SSBs had a six- to 12-fold higher odds of obesity (aOR 6.4, 95\% CI:2.4, 17.2 for fruit juice; aOR 6.6, 95\% CI:2.7, 14.8 for fruit juice or SSBs; and aOR 12.2, 95\% CI:4.3, 25 for SSBs; all $p<0.001)$. Compared with children with adequate BF and no intake of SSB or $100 \%$ fruit juice, those with adequate BF and intake of 100\% fruit juice and/or SSBs had a threefold higher odds of obesity (aOR 3.1, 95\% CI:1.1, 7.3 for fruit juice; aOR 3.3, 95\% CI:1.3, 8.3 for fruit juice or SSBs; and aOR 3.4, 95\% CI:1.3, 8.5 for SSBs; all $p<0.05$ ), while those with inadequate BF ( $<6$ months) and intake of $100 \%$ fruit juice and/or SSB were associated with five- to tenfold higher odds of obesity (aOR 4.8, 95\% CI:2.3, 12.2 for fruit juice; aOR 6.0, 95\% CI:2.5, 12.8 for fruit juice or SSBs; aOR 9.5, 95\% CI:3.7, 15.1 for SSBs; all $p<0.05$ ).

Conclusions/interpretation This is the first study to prospectively evaluate the relation of BF or EBF duration and intake of SSB and $100 \%$ fruit juice during the first year of life with subsequent obesity in children exposed to GDM. Adequate BF or EBF combined with avoidance of SSB and 100\% fruit juice during early infancy may ameliorate future child obesity in this high-risk population.
\end{abstract}

Keywords $100 \%$ fruit juice $\cdot$ BMI $\cdot$ Childhood obesity $\cdot$ Exclusive breastfeeding $\cdot$ Gestational diabetes mellitus $\cdot$ Infant diet . Sugar-sweetened beverages

Sarvenaz Vandyousefi

Sarvenaz.Vandyousefi@nyulangone.org

Erica P. Gunderson

erica.gunderson@kp.org

1 Department of Pediatrics, New York University Grossman School of Medicine, New York, NY, USA

2 Department of Nutritional Sciences, University of Texas at Austin, Dell Pediatrics Research Institute, Austin, TX, USA

3 Division of Research, Cardiovascular and Metabolic Conditions Section, Kaiser Permanente Northern California, Oakland, CA, USA

$\begin{array}{ll}\text { Abbreviations } \\ \text { AAP } & \text { The American Academy of Pediatrics } \\ \text { AGA } & \text { Appropriate for gestational age } \\ \text { BF } & \text { Breastfeeding } \\ \text { EBF } & \text { Exclusive breastfeeding } \\ \text { EHR } & \text { Electronic health record } \\ \text { FF } & \text { Formula feeding } \\ \text { GDM } & \text { Gestational diabetes mellitus } \\ \text { KPNC } & \text { Kaiser Permanente Northern California } \\ \text { LGA } & \text { Large for gestational age }\end{array}$




\section{Research in context}

\section{What is already known about this subject?}

- Gestational diabetes mellitus (GDM) increases obesity risk in offspring

- Breastfeeding is a recommended infant feeding behaviour that is associated with decreased risk of obesity later in the child's life

- Much less is known about the impact of naturally sweetened fruit juice and sugary beverage intake during early infancy on the risk of obesity in children exposed to GDM

\section{What is the key question?}

- $\quad$ Are there independent and joint associations between adequate 'any' breastfeeding (BF) or exclusive breastfeeding (EBF), intake of sugar-sweetened beverages and $100 \%$ fruit juice during the first year of life and subsequent overweight and obesity among children (aged 2-5 years) exposed to GDM?

\section{What are the new findings?}

- Inadequate duration of BF and EBF ( $<6$ months) were each directly associated with child obesity in GDM-exposed children at 2-5 years of age

- $\quad$ Sugar-sweetened beverage and fruit juice intake in the first year were each independently associated with a higher odds of child obesity in GDM-exposed children at 2-5 years of age

- Inadequate EBF combined with intake of fruit juice or sugar-sweetened beverages before 1 year of age was associated with six- and 12-fold higher odds of obesity, respectively, in GDM-exposed children at 2-5 years of age. There were similar associations for adequate $\mathrm{EBF}$ and $\mathrm{BF}$ and inadequate $\mathrm{BF}$, when combined with intake of fruit juice and sugar-sweetened beverages

How might this impact on clinical practice in the foreseeable future?

- These results support the implementation of early life behavioural interventions to counter obesogenic feeding practices during infancy in populations at higher risk of obesity and diabetes

SGA Small for gestational age

SSB Sugar-sweetened beverage

SWIFT Study of Women, Infant Feeding and Type 2 Diabetes after GDM Pregnancy

U.S. United States

WIC Special Supplemental Nutrition Program for Women, Infants and Children

\section{Introduction}

Intrauterine exposure to maternal glucose intolerance, known as gestational diabetes mellitus (GDM), is one of the most common metabolic complications of pregnancy, affecting 2$10 \%$ of all pregnant women annually in the USA [1] and up to $28 \%$ worldwide depending on population characteristics [2]. GDM increases the risk of many adverse maternal and perinatal health outcomes. Children exposed to GDM are at higher risk of macrosomia and being born large for gestational age (LGA), and have an increased lifelong risk of obesity [3]. In 2015-2016, the prevalence of overweight and obesity among U.S. children and adolescents (aged 2-19 years) were 16.6\% and $18.5 \%$, respectively; while obesity prevalence was about $14 \%$ among children aged $2-5$ years $[4,5]$. GDM and early life dietary behaviours such as limited breastfeeding (BF), limited exclusive $\mathrm{BF}(\mathrm{EBF})$ and other postnatal dietary habits may influence weight gain, obesity and metabolic disease among young children and adolescents [6-11]. Prospective studies of GDM infants show conflicting findings for BF measures and infant linear ponderal growth. In South Asian infants exposed to GDM, BF duration was associated with accelerated growth compared with formula fed infants, but perinatal outcomes including prematurity were not evaluated in this study [12]. In contrast, our analysis of 464 motherinfant dyads exposed to GDM showed that higher intensity and duration of BF were inversely associated with infant ponderal growth, weight gain, and change in weight-forlength $z$ scores using repeated measurements from birth and through the first year [7]. This analysis accounted for maternal pre-pregnancy BMI, gestational weight gain, sociodemographics, GDM severity, newborn outcomes including gestational age, infant sleep and complementary feeding.

Early introduction to complementary foods, SSBs and unsweetened fruit juice have been directly associated with obesity in young children [8,13-15]. The American Academy of Pediatrics (AAP) recommends that breast milk be the sole source of nutrients during the infant's first 6 months 
of life and that solid foods or liquids be introduced around 6 months of age [16]. The AAP also recommends avoiding fruit juice and SSBs during the infant's first year because they have a high sugar content, lower nutrient content, and links with obesity [17]. Studies of GDM-exposed children were unable to evaluate infant dietary intake, except for breast and formula feeding (FF). This is because most of them had retrospective designs that looked at older children and asked mothers to recall $\mathrm{BF}$ duration and whether $\mathrm{BF}$ was mixed with formula [10, 18-21]; one prospective study has been conducted, but it had a small sample size $(n<58)$ [22]. Other studies have evaluated cross-sectional associations between concurrent intake of SSBs or fruit juice with child obesity (ages 5-16 years) and GDM exposure [8, 13-15, 23, 24]. In contrast, the Study of Women, Infant Feeding and Type 2 Diabetes after GDM Pregnancy (SWIFT) enrolled 1035 mother-infant dyads in which the mother had GDM, and prospectively assessed both $\mathrm{BF}$ intensity and duration and infant dietary intake (e.g. SSBs, fruit juice, artificial formula, other) every month from birth to 1 year of age [25].

In this study, we evaluated the independent and joint associations between BF measures and infant dietary intake and child BMI percentile categories at 2-5 years of age among GDM-exposed children. We hypothesised that higher BF intensity and longer BF duration, and avoidance of SSBs and $100 \%$ fruit juice intake during the first year of life are associated with lower risk of overweight or obesity at 25 years of age among children exposed to GDM, after accounting for the intrauterine milieu (maternal BMI, glucose intolerance, GDM treatment and gestational weight gain), sociodemographics and other factors known to affect child obesity.

\section{Methods}

Study sample: mother-infant dyads This analysis utilises data from the SWIFT study, which is a prospective, longitudinal study of 1035 women with recent GDM enrolled into inperson research examinations from September 2008 to December 2011. The study eligibility criteria were as follows: (1) Kaiser Permanente Northern California (KPNC) member delivered at a Kaiser Permanente hospital (2008-2011), (2) maternal age 20-45 years at delivery, (3) diagnosed with GDM according to the $3 \mathrm{~h} 100 \mathrm{~g}$ OGTT per Carpenter and Coustan criteria [26], (4) delivery of a singleton, live birth at $\geq 35$ weeks gestation, (5) no history of overt diabetes or other serious medical conditions, (6) electronic health record (EHR) data for the GDM pregnancy, (7) not planning to become pregnant again or move out of the area in the next 2 years, (8) not taking any medications that affect blood glucose tolerance, (9) able to speak English or Spanish, and (10) exclusive or mostly BF, or exclusive or mostly FF and the intention to continue for $\geq 4$ months as detailed in the SWIFT study design $[25,27]$.

Briefly, the study contacted women with GDM during late pregnancy and again at 4 weeks postpartum to evaluate $\mathrm{BF}$ intention and study eligibility. The SWIFT study enrolled 1035 women within a single integrated healthcare system who provided written informed consent for three in-person research visits starting from 6 to 9 weeks postpartum (study baseline) and annually thereafter for 2 years. In this analysis, 835 of 1031 SWIFT mother-infant dyads exposed to GDM and continued in study follow-up had complete information on BF and infant dietary intake variables (from birth to 1 year), sociodemographics, prenatal and newborn clinical outcomes, child weight and height measurements at 2-5 years of age, and mothers did not have overt diabetes at study baseline. Those excluded from the analytic sample $(n=196)$ had lower attained education, worse maternal glucose intolerance and shorter BF duration than women included (electronic supplementary material (ESM) Table 1).

Data collection At each SWIFT study in-person research visit for women (6-9 weeks postpartum, 1 year and 2 year postbaseline), trained research staff conducted a 2 h 75 g OGTT (glucose and insulin) and obtained measurements of maternal anthropometry (weight, height, waist circumference), and administered interviewer- and self-administered surveys to assess reproductive and health history, infant feeding/diet, child health, sociodemographic factors (race/ethnicity, education, income), depression and maternal lifestyle behaviours (diet, physical activity, sleep). Participants also completed monthly mailed surveys from delivery to 1 year later to report BF intensity and duration, health status, and detailed infant dietary intake [25].

This study also obtained clinical data from the Kaiser Permanente EHR to determine study eligibility and account for prenatal exposure to maternal hyperglycaemia, including GDM diagnosis and treatment (i.e. prenatal 3 h 100 g OGTT, gestational age at GDM diagnosis, and GDM treatment with diet, oral medication, and/or insulin therapy), maternal height, pre-pregnancy weight and last weight measured before delivery (gestational weight gain). Data on newborn outcomes (i.e. infant sex, gestational age, birthweight, weight and length for size-at-birth percentiles) and child weight and height measurement between the ages of 2 and 5 years were obtained from the EHR. The SWIFT Study protocols were approved by the Kaiser Permanente Northern California institutional review board and were carried out in accordance with the Declaration of Helsinki as revised in 2008.

Infant feeding and dietary intake measures Telephone interviews were conducted during late pregnancy to assess $\mathrm{BF}$ intention and again at 4-6 weeks post-delivery to assess BF intensity and intention to assess eligibility for enrolment. 
Women completed detailed infant feeding surveys at inperson research visits up to 2 years, a telephone interview at 6 months, and monthly mailed surveys from birth to 12 months. Women reported the average frequency of BF and FF within the past 7 days for each month, including total number of all feedings, BF and daily amounts of formula fed, as well as the date of introduction of solids, fruit juice, sweetened beverages, water, sugar water, and other beverages, including types and amounts of each item. BF duration (total months of any $\mathrm{BF}$ ) was evaluated as a continuous variable, and dichotomised as adequate $\mathrm{BF}$ ( $\geq 6$ months) or inadequate $\mathrm{BF}$ (none or $<6$ months). EBF duration was dichotomised as adequate $\mathrm{EBF}$ ( $\geq 6$ months) or inadequate EBF (none or $<6$ months). The SWIFT study utilised the method of Piper and Parks [28] to quantitatively assess BF intensity and duration [7]. This method calculates a BF intensity score for each month by dividing the number of breast milk feeds by the total number of feeds (FF, BF, other liquids) in $24 \mathrm{~h}$ (on average during the past 7 days). The monthly score ranges from 0 for exclusive FF to 1 for EBF. The 12 month BF intensity/ duration score is the sum of the 12 intensity ratios from birth to 12 months (range of scores: 0 to 12), as a single continuous measure across time.

The infant diet variables were assessed from birth to 12 months, including age at initiation of first complementary foods, intake of fruit juice (100\% fruit juice or juice with added sugar), liquids with added sugar, and SSBs. Intake of beverages other than milk feeds during the first year of life were categorised as: (1) consuming SSBs (including any sugary drinks, Pedialyte, water or juices with added sugar), (2) $100 \%$ fruit juice intake (unsweetened/with no added sugar), or (3) no $\mathrm{SSB} /$ no $100 \%$ fruit juice from birth to 1 year of age. Other food items were assessed and included in the complementary food intake variable categorised by age of initiation.

\section{Clinical anthropometric measurements from the EHR at birth}

and 2-5 years Clinical measurements of neonatal weight and length in the supine position were obtained from the EHR to calculate birthweight percentiles based on the KPNC population $[29,30]$. We also obtained child weight and height from the EHR clinical paediatric well check visits (measured height in a standing position without shoes and heels against a wall using a stadiometer, and measured weight using a calibrated digital scale) between 2 and 5 years of age [31, 32]. For this analysis, the weight and height measured at the oldest age between 2 and 5 years for each child was selected to calculate the standardised BMI percentiles for age and sex, and categorise each child as normal (BMI $<85$ th percentile), overweight (BMI $\geq 85$ th to $<95$ th percentile) or obese (BMI $\geq 95$ th percentile) based on the Centers for Disease Prevention and Control growth percentiles [33]. ESM Table 2 displays per cent of individuals among the BMI percentile categories by child age at last weight and height measurements.
Data analysis Descriptive statistics (i.e. mean, SD, range, median and quartiles, histograms and Q-Q plots) assessed the variable distributions. Four separate linear and multinomial logistic regression models estimated adjusted OR (aOR) for the following BF measures: (1) the continuous BF duration, (2) any BF duration categories, (3) EBF duration categories, and (4) 12 month BF intensity/duration score, as well as SSB intake for child BMI percentile categories at ages 2-5 years. For the current analysis, a priori covariates were selected for their known influence on child obesity, including maternal education level (years of formal schooling), gestational weight gain, parity (primiparous vs multiparous), Special Supplemental Nutrition Program for Women, Infants and Children (WIC) participation, infant sex, size for gestational age, race/ethnicity, and child age at BMI measurement. Prenatal GDM severity was measured according to three variables: (1) $3 \mathrm{~h} 100 \mathrm{~g}$ OGTT laboratory results calculated as the sum of four $z$ scores for glucose, (2) GDM treatment type (diet only vs oral hypoglycaemic agents or insulin), and (3) gestational age at GDM diagnosis.

Finally, to evaluate the joint association between the infant dietary intake categories (SSB, and 100\% fruit juice) and categorical $\mathrm{BF}$ variables, two separate multinomial regression models were constructed, each stratified by BF adequacy and EBF adequacy adjusted for the above covariates. All analyses were performed with SAS version 9.4 (SAS, North Carolina, USA). Significance was denoted at $p<0.05$.

\section{Results}

Descriptive statistics and correlates Of 835 mother-infant dyads, BMI percentiles were obtained for $90 \%$ of children using weight and height measured at 3 years or older, with a mean \pm SD age at the last BMI percentile of $40.5 \pm 6.3$ months. Regarding race/ethnicity, $37 \%$ of children were Non-Hispanic Asian, $23 \%$ were Non-Hispanic White, $8 \%$ were Black and $30 \%$ were of Hispanic origin. Maternal age, GDM treatment type, gestational weight gain, and parity did not differ among child BMI categories (Table 1). Child obesity was associated with higher maternal pre-pregnancy BMI and prenatal $3 \mathrm{~h}$ $100 \mathrm{~g}$ OGTT $z$ score sum, lower education, earlier gestational age at GDM diagnosis and WIC participation (Table 1).

Birth outcomes, infant diet and other mother-infant dyad characteristics among the child BMI percentile categories at age $2-5$ years are shown in Table 2 . Thirteen per cent $(n=$ 106) of children were classified with overweight, and $11 \%$ $(n=86)$ with obesity. Child age at BMI, sex or age of initiation of complementary foods were not associated with BMI percentile categories. Children with obesity were more likely to have been LGA than those with overweight and normal weight. Compared with normal weight children, those with overweight and obesity had significantly higher mean 
Table 1 Maternal characteristics among BMI percentile categories for young children aged 2 to 5 years exposed to GDM

\begin{tabular}{|c|c|c|c|c|c|}
\hline \multirow[t]{2}{*}{ Characteristics $^{\mathrm{a}}$} & \multicolumn{5}{|c|}{ Child BMI percentile categories at ages 2 to 5 years $^{\mathrm{b}}$} \\
\hline & $\begin{array}{l}\text { All } \\
n=835\end{array}$ & $\begin{array}{l}\text { Normal } \\
n=643\end{array}$ & $\begin{array}{l}\text { Overweight } \\
n=106\end{array}$ & $\begin{array}{l}\text { Obesity } \\
n=86\end{array}$ & $p$-value ${ }^{\mathrm{c}}$ \\
\hline Maternal age (years) & $33.4 \pm 4.8$ & $33.5 \pm 4.8$ & $33.1 \pm 4.5$ & $32.7 \pm 5.5$ & 0.29 \\
\hline Education (years) & $14.9 \pm 2.9$ & $15.1 \pm 2.9$ & $14.6 \pm 2.6$ & $13.6 \pm 2.7$ & $<0.001$ \\
\hline \multicolumn{6}{|l|}{ Race/ethnicity, $n(\%)$} \\
\hline Non-Hispanic White & $195(23)$ & $149(23)$ & $29(27)$ & $17(20)$ & \multirow[t]{5}{*}{$<0.01$} \\
\hline Non-Hispanic Black & $67(8)$ & $46(7)$ & $8(8)$ & $13(15)$ & \\
\hline Non-Hispanic Asian & $311(37)$ & $262(41)$ & $28(26)$ & $21(24)$ & \\
\hline Hispanic & $246(30)$ & $172(27)$ & $39(37)$ & $35(41)$ & \\
\hline Mixed race/other & $16(2)$ & $14(2)$ & $2(2)$ & $0(0)$ & \\
\hline WIC recipient, $n(\%)$ & $211(25)$ & $142(22)$ & $29(27)$ & $40(47)$ & $<0.001$ \\
\hline Pre-pregnancy BMI $\left(\mathrm{kg} / \mathrm{m}^{2}\right)$ & $29.5 \pm 7.3$ & $28.5 \pm 6.8$ & $31.7 \pm 8.3$ & $33.6 \pm 7.1$ & $<0.001$ \\
\hline Prenatal 3 h 100 g OGTT $^{d} z$ score sum & $0 \pm 2.7$ & $-0.1 \pm 2.5$ & $-0.3 \pm 2.4$ & $0.9 \pm 3.6$ & $<0.01$ \\
\hline \multicolumn{6}{|l|}{ GDM treatment type, $n(\%)$} \\
\hline Diet only & $587(70)$ & $457(71)$ & $74(70)$ & $56(65)$ & \multirow[t]{3}{*}{0.45} \\
\hline Oral hypoglycaemic agents & $223(27)$ & $165(26)$ & $29(27)$ & $29(34)$ & \\
\hline Insulin & $25(3)$ & $21(3)$ & $3(3)$ & $1(1)$ & \\
\hline Gestational age at GDM ${ }^{\mathrm{e}}$ (weeks) & $28(25,29)$ & $28(26,29)$ & $27(25,29)$ & $27(13,29)$ & $<0.01$ \\
\hline Gestational weight gain (kg) & $10.5 \pm 6.8$ & $10.6 \pm 6.3$ & $10.2 \pm 9.1$ & $9.9 \pm 7.7$ & 0.65 \\
\hline \multicolumn{6}{|l|}{ Parity birth order, $n(\%)$} \\
\hline Primiparous & $319(38)$ & $238(37)$ & $48(45)$ & $33(38)$ & \multirow[t]{2}{*}{0.08} \\
\hline Multiparous & $516(62)$ & $405(63)$ & $58(55)$ & $53(62)$ & \\
\hline \multicolumn{6}{|c|}{${ }^{\mathrm{a}}$ Values are mean \pm SD unless otherwise stated } \\
\hline \multirow{2}{*}{\multicolumn{6}{|c|}{$\begin{array}{l}{ }^{\mathrm{b}} \text { Normal: BMI-for-age }<85 \text { th percentile. Overweight: BMI-for-age } 85 \text { th to }<95 \text { th percentile. Obesity: BMI-for- } \\
\text { age } \geq 95 \text { th percentile } \\
\text { c } p \text { values }<0.05 \text { are significant; } t \text { tests and } \chi^{2} \text { tests were run to assess difference in means or } \% \text { between BMI } \\
\text { percentile groups }\end{array}$}} \\
\hline & & & & & \\
\hline \multicolumn{6}{|c|}{$\begin{array}{l}{ }^{\mathrm{d}} \text { Prenatal } 3 \mathrm{~h} 100 \mathrm{~g} \text { OGTT sum of four } z \text { scores }(z \text { score for the glucose value at each time point; GDM severity) } \\
{ }^{\mathrm{e}} \text { Gestational age at GDM diagnosis [median (Q1, Q3)] }\end{array}$} \\
\hline
\end{tabular}

birthweight and birth length. All 835 children were exposed to GDM but only $20 \%(n=163)$ were exclusively breastfed for 6 months (adequate EBF). Fifty-one per cent $(n=424)$ consumed $100 \%$ juice before 1 year of age, and $16 \%(n=$ 131) consumed SSB before 1 year of age. Compared with children with normal weight and overweight, children with obesity had less favourable infant diet intake including shorter mean BF duration (9.4 and 8.5 vs 6.4 months; $p<0.001$ ), lower proportions having adequate $\mathrm{BF}$ duration (BF $\geq 6$ months; $62 \%$ and $59 \%$ vs $42 \% ; p<0.01)$ or adequate EBF $(21 \%$ and $19 \%$ vs $12 \% ; p<0.01)$, and were more likely to have consumed $100 \%$ fruit juice $(57 \%)$ and SSBs $(26 \%)$ during the first year of life $(p<0.01)$. Early introduction to complementary food ( $\leq 4$ and $\leq 6$ months of age) was not associated with child overweight or obesity at $2-5$ years of age.

Multinomial logistic and linear regression analyses Compared with adequately breastfed infants (any BF or EBF for $\geq 6$ months), infants who were inadequately breastfed were more likely to consume SSBs or $100 \%$ fruit juice in the first year of life (ESM Table 3). The multiple linear regression model showed an inverse association between months of any BF duration and BMI percentile adjusted for all covariates ( $\mathrm{B}=-0.009,95 \% \mathrm{CI}:-0.02,-0.003, p=0.003)$. Results from multinomial logistic regression models adjusted for covariates are shown in Table 3. BF duration, EBF duration and 12 month BF intensity/duration scores were each inversely associated with odds of child obesity at 2-5 years of age. Compared with children with adequate $\mathrm{BF}$, inadequate $\mathrm{BF}$ was associated with a twofold higher odds of obesity. Similar strength of direct associations were found between inadequate $\mathrm{EBF}$ ( $<6$ months), and 12 month BF intensity/ duration score ( $<6$ months) with odds of child obesity. However, there were no significant associations between $\mathrm{BF}, \mathrm{EBF}$ and $\mathrm{BF}$ intensity/duration score categories for child overweight compared with normal weight. Infant beverage intake was associated with child BMI percentile categories. Both SSBs and $100 \%$ juice intakes before age 1 year were 
Table 2 Characteristics at birth and infant feeding during the first year of life associated with overweight and obesity categories among children aged 2 to 5 years exposed to GDM

\begin{tabular}{|c|c|c|c|c|c|}
\hline \multirow[t]{2}{*}{ Characteristics $^{\mathrm{a}}$} & \multicolumn{5}{|c|}{ Child BMI categories at ages 2 to 5 years ${ }^{\mathrm{b}}$} \\
\hline & $\begin{array}{l}\text { All } \\
n=835\end{array}$ & $\begin{array}{l}\text { Normal } \\
n=643\end{array}$ & $\begin{array}{l}\text { Overweight } \\
n=106\end{array}$ & $\begin{array}{l}\text { Obesity } \\
n=86\end{array}$ & $p$-value \\
\hline Male sex, $n(\%)$ & $433(52)$ & $331(51)$ & $52(49)$ & $50(58)$ & 0.42 \\
\hline Birthweight (g) & $3397 \pm 499$ & $3341 \pm 484$ & $3531 \pm 506$ & $3621 \pm 478$ & $<0.001$ \\
\hline Birth length $(\mathrm{cm})$ & $50.5 \pm 2.4$ & $50.4 \pm 2.5$ & $50.8 \pm 2.3$ & $50.9 \pm 2.3$ & 0.01 \\
\hline Gestational age (weeks) & $39 \pm 1.2$ & $39 \pm 1.2$ & $39.2 \pm 1$ & $39.1 \pm 1.2$ & 0.21 \\
\hline \multicolumn{6}{|l|}{ Size-at-birth percentile } \\
\hline LGA & $176(21)$ & $113(18)$ & $27(25)$ & $36(42)$ & \multirow[t]{3}{*}{$<0.001$} \\
\hline AGA & $641(77)$ & $513(80)$ & $78(74)$ & $50(56)$ & \\
\hline SGA & $18(2)$ & $17(3)$ & $1(1)$ & $0(0)$ & \\
\hline Child age at follow-up BMI (months) & $40.5 \pm 6.2$ & $40.5 \pm 6.2$ & $40.5 \pm 6.1$ & $40.9 \pm 6.4$ & 0.56 \\
\hline \multicolumn{6}{|l|}{ Infant dietary intake } \\
\hline Total BF (any) duration (months) & $9 \pm 7.2$ & $9.4 \pm 7.3$ & $8.5 \pm 7.4$ & $6.4 \pm 6.2$ & $<0.001$ \\
\hline \multicolumn{6}{|l|}{$\mathrm{BF}$ duration categories } \\
\hline Inadequate BF (<6 months) & $335(40)$ & $242(38)$ & $43(41)$ & $50(58)$ & \multirow[t]{2}{*}{$<0.01$} \\
\hline Adequate $\mathrm{BF}$ ( $\geq 6$ months) & $500(60)$ & $401(62)$ & $63(59)$ & $36(42)$ & \\
\hline \multicolumn{6}{|l|}{ EBF duration categories } \\
\hline Inadequate $\mathrm{EBF}$ ( $<6$ months) & $672(80)$ & $510(79)$ & $86(81)$ & $76(88)$ & \multirow[t]{2}{*}{$<0.01$} \\
\hline Adequate EBF ( $\geq 6$ months) & $163(20)$ & $133(21)$ & $20(19)$ & $10(12)$ & \\
\hline \multicolumn{6}{|l|}{ BF intensity groups at age $6-9$ weeks } \\
\hline Exclusively BF & $183(22)$ & $149(23)$ & $25(23)$ & $9(11)$ & \multirow[t]{4}{*}{0.04} \\
\hline Mostly BF & $342(41)$ & $269(42)$ & $41(39)$ & $32(36)$ & \\
\hline Mixed/inconsistent FF & $176(21)$ & $129(20)$ & $22(21)$ & $25(27)$ & \\
\hline Exclusively or mostly FF & $134(16)$ & $96(15)$ & $18(17)$ & $20(26)$ & \\
\hline \multicolumn{6}{|l|}{$\mathrm{BF}$ intensity and duration categories ${ }^{\mathrm{d}}$} \\
\hline 12 month score $<6$ & $440(53)$ & $317(49)$ & $62(58)$ & $61(71)$ & \multirow[t]{2}{*}{$<0.001$} \\
\hline 12 month score $\geq 6$ & $395(47)$ & $326(51)$ & $44(42)$ & $25(29)$ & \\
\hline \multicolumn{6}{|l|}{ Beverage intake from birth to 1 year } \\
\hline No SSB/no $100 \%$ fruit juice & $280(33)$ & $232(36)$ & $33(31)$ & $15(17)$ & \multirow[t]{3}{*}{$<0.01$} \\
\hline $100 \%$ fruit juice & $424(51)$ & $319(50)$ & $56(53)$ & $49(57)$ & \\
\hline SSB & $131(16)$ & $92(14)$ & $17(16)$ & $22(26)$ & \\
\hline \multicolumn{6}{|c|}{ Complementary feeding age of initiation categories } \\
\hline$\leq 4$ months (early) & $136(26)$ & $100(16)$ & $17(16)$ & $19(22)$ & \multirow[t]{2}{*}{0.37} \\
\hline$>4$ months (later) & $699(84)$ & $543(84)$ & $89(84)$ & $67(78)$ & \\
\hline$\leq 6$ months (early) & $647(77)$ & $495(77)$ & $82(77)$ & $70(73)$ & 0.57 \\
\hline$>6$ months (later) & $188(23)$ & $148(23)$ & $24(23)$ & $16(27)$ & \\
\hline
\end{tabular}

${ }^{\text {a }}$ Values are mean \pm SD unless otherwise stated

${ }^{\mathrm{b}}$ Normal: BMI-for-age $<85$ th percentile. Overweight: BMI-for-age 85 th to $<95$ th percentile. Obesity: BMI-forage $\geq 95$ th percentile

${ }^{c} p$ values $<0.05$ are significant; $t$ tests and $\chi^{2}$ tests were run to assess difference in means or $\%$ between BMI percentile groups

${ }^{\mathrm{d}}$ Overall score sum of 12 monthly ratios (birth to 12 months of age; range $0-12$ ) each associated with higher odds of child obesity at $2-5$ years independent of BF duration (SSB aOR 3.22, 95\% CI:1.55, $6.67, p<0.01$, and $100 \%$ fruit juice, aOR $2.18,95 \%$ CI:1.17, 4.06, $p=0.01)$. However, these associations were null for the odds of overweight.
In the models stratified by adequate BF and EBF categories and infant beverage categories, there were overall associations with the odds of child overweight and obesity in the adjusted model. Compared with children with adequate $\mathrm{BF}$ and no SSBs or $100 \%$ juice intake during infancy (referent), those 
Table 3 Logistic multinomial regression for infant feeding and covariables during the first year of life and the adjusted odds ratios with $95 \%$ CIs for overweight and obesity among children aged 2 to 5 years exposed to GDM

\begin{tabular}{|c|c|c|c|c|c|}
\hline \multirow[t]{3}{*}{ Variable } & \multicolumn{5}{|c|}{ Child BMI categories at ages 2 to 5 years $^{\mathrm{a}}$} \\
\hline & \multicolumn{2}{|l|}{ Overweight } & \multicolumn{2}{|l|}{ Obesity } & \multirow[b]{2}{*}{ Overall $p$ value $^{\mathrm{c}}$} \\
\hline & $\mathrm{aOR}^{\mathrm{b}}(95 \% \mathrm{CI})$ & $p$ value $^{\mathrm{c}}$ & $\mathrm{aOR}^{\mathrm{b}}(95 \% \mathrm{CI})$ & $p$ value ${ }^{\mathrm{c}}$ & \\
\hline Model 1: Any breastfeeding duration (BF) categories & & & & & 0.02 \\
\hline Adequate $\mathrm{BF}$ ( $\geq 6$ months) & 1.00 & Referent & 1.00 & ----- & \\
\hline Inadequate $\mathrm{BF}$ ( $<6$ months $)$ & $1.10(0.72,1.68)$ & 0.97 & $1.99(1.23,3.21)$ & 0.01 & \\
\hline Model 2: Exclusive BF duration (EBF) categories & & & & & $<0.01$ \\
\hline Adequate EBF ( $\geq 6$ months) & 1.00 & Referent & 1.00 & ---- & \\
\hline Inadequate EBF ( $<6$ months) & $1.04(0.68,1.59)$ & 0.86 & $2.11(1.32,3.35)$ & $<0.01$ & \\
\hline \multicolumn{6}{|l|}{ Model 3: BF intensity/duration score ${ }^{\mathrm{d}}$ categories } \\
\hline 12 month score $\geq 6$ & 1.00 & Referent & 1.00 & ----- & $<0.01$ \\
\hline 12 month score $<6$ & $1.44(0.95,2.19)$ & 0.09 & $2.21(1.33,3.67)$ & $<0.01$ & \\
\hline \multicolumn{6}{|l|}{ Covariates for model 1 (above) ${ }^{\mathrm{e}}$} \\
\hline Beverage feeding (intake from birth to 1 year) & & & & & 0.03 \\
\hline No SSB/no $100 \%$ fruit juice & 1.00 & Referent & 1.00 & ----- & \\
\hline $100 \%$ fruit juice & $1.24(0.78,1.98)$ & 0.48 & $2.18(1.17,4.06)$ & 0.02 & \\
\hline SSB & $1.31(0.69,2.50)$ & 0.67 & $3.22(1.55,6.67)$ & $<0.01$ & \\
\hline Race/ethnicity, $n(\%)$ & & & & & 0.03 \\
\hline Non-Hispanic White & 1.00 & Referent & 1.00 & ---- & \\
\hline Hispanic & $1.54(1.03,2.38)$ & 0.04 & $1.61(1.05,2.63)$ & 0.03 & \\
\hline Prenatal 3 h 100 g OGTT sum of $4 z$ scores & $0.95(0.87,1.03)$ & 0.21 & $1.08(1.00,1.16)$ & 0.04 & 0.04 \\
\hline Size-at-birth percentile categories & & & & & $<0.001$ \\
\hline AGA or SGA & 1.00 & Referent & 1.00 & ----- & \\
\hline LGA & $1.33(0.80,2.18)$ & 0.26 & $2.68(1.62,4.41)$ & $<0.001$ & \\
\hline Maternal gestational weight gain $(\mathrm{kg})$ & $0.97(0.96,1.02)$ & 0.55 & $0.97(0.94,1.01)$ & 0.14 & 0.80 \\
\hline Maternal pre-pregnancy BMI (kg/m²) & $1.06(1.03,1.09)$ & $<0.001$ & $1.07(1.03,1.10)$ & $<0.0001$ & $<0.0001$ \\
\hline Gestational age at GDM diagnosis (weeks) & $1.01(0.98-1.04)$ & 0.41 & $0.97(0.94,0.99)$ & 0.03 & 0.04 \\
\hline Model 4: Any BF-beverage categories & & & & & 0.03 \\
\hline Adequate $\mathrm{BF}$, no $\mathrm{SSB} / 100 \%$ juice intake & 1.00 & Referent & 1.00 & ---- & \\
\hline Adequate $\mathrm{BF}$, and $100 \%$ fruit juice & $1.23(0.73,2.34)$ & 0.65 & $3.13(1.11,7.29)$ & 0.03 & \\
\hline Adequate BF, and SSB or $100 \%$ fruit juice & $1.28(0.71,2.18)$ & 0.38 & $3.32(1.29,8.25)$ & $<0.01$ & \\
\hline Adequate BF, and SSB & $1.31(0.51,2.94)$ & 0.65 & $3.35(1.32,8.50)$ & 0.01 & \\
\hline Inadequate $\mathrm{BF}$, no $\mathrm{SSB} / 100 \%$ juice intake & $1.16(0.53,2.53)$ & 0.71 & $3.25(1.29,9.62)$ & 0.03 & \\
\hline Inadequate $\mathrm{BF}$, and $100 \%$ fruit juice & $1.31(0.73,2.35)$ & 0.36 & $4.78(2.33,12.19)$ & $<0.01$ & \\
\hline Inadequate $\mathrm{BF}$, and $\mathrm{SSB}$ or $100 \%$ fruit juice & $1.32(0.64,2.12)$ & 0.61 & $6.03(2.45,12.80)$ & $<0.001$ & \\
\hline Inadequate BF, and SSB & $1.60(0.68,3.76)$ & 0.27 & $9.64(3.70,15.10)$ & $<0.0001$ & \\
\hline Model 5: EBF-beverage categories & & & & & $<0.01$ \\
\hline Adequate EBF, no SSB/100\% juice intake & 1.00 & Referent & 1.00 & ----- & \\
\hline Adequate EBF, and $100 \%$ fruit juice & $1.28(0.73,2.27)$ & 0.39 & $4.17(1.55,11.17)$ & $<0.01$ & \\
\hline Adequate $\mathrm{EBF}$, and $\mathrm{SSB}$ or $100 \%$ fruit juice & $1.05(0.55,1.99)$ & 0.43 & $4.45(1.40,8.47)$ & $<0.01$ & \\
\hline Adequate $\mathrm{EBF}$, and SSB & $1.26(0.73,2.18)$ & 0.72 & $4.71(1.43,15.01)$ & $<0.01$ & \\
\hline Inadequate $\mathrm{EBF}$, no $\mathrm{SSB} / 100 \%$ juice intake & $1.07(0.48,2.37)$ & 0.57 & $4.43(1.43,13.71)$ & $<0.01$ & \\
\hline Inadequate $\mathrm{EBF}$, and $100 \%$ fruit juice & $1.22(0.65,2.29)$ & 0.53 & $6.40(2.38,17.16)$ & $<0.001$ & \\
\hline Inadequate $\mathrm{EBF}$, and $\mathrm{SSB}$ or $100 \%$ fruit juice & $1.29(0.72,2.31)$ & 0.75 & $6.60(2.71,14.85)$ & $<0.001$ & \\
\hline Inadequate $\mathrm{EBF}$, and $\mathrm{SSB}$ & $1.50(0.65,3.48)$ & 0.34 & $12.28(4.26,25.40)$ & $<0.0001$ & \\
\hline
\end{tabular}

${ }^{\text {a }}$ Overweight: BMI-for-age 85 th to $<95$ th percentile; obesity: BMI-for-age $>95$ th percentile

$\mathrm{b}$ aOR values

${ }^{\mathrm{c}} p$ values $<0.05$ are significant

${ }^{\mathrm{d}}$ Overall BF score sum of 12 monthly ratios (birth to 12 months of age; range 0-12)

${ }^{\mathrm{e}}$ Covariates are from the regression model with any BF variable

The following covariates were used in all five regression models: maternal education level, race/ethnicity, gestational weight gain, prenatal $3 \mathrm{~h} 100 \mathrm{~g}$ OGTT sum of $4 z$ scores ( $z$ score for glucose values at each time point; GDM severity), GDM treatment type, gestational age at GDM diagnosis, WIC participation, parity, and infant sex, infant size for gestational age and sex at birth and age at BMI measurement 
with adequate $\mathrm{BF}$ and $100 \%$ fruit juice intake, SSB intake, or both combined from birth to 1 year of age had threefold higher odds of obesity (all $p<0.05$ ). Figure 1 displays the aOR of obesity by BF-beverage groups among the children (25 years), of whom all were exposed to GDM.

Similarly, compared with children with adequate EBF and no SSB or $100 \%$ fruit juice intake during the first year (referent), those with adequate EBF and $100 \%$ fruit juice, SSBs intake or both combined had 4.2- to 4.7fold higher odds of obesity. Compared with the referent group, children with inadequate $\mathrm{BF}$ and no SSBs or $100 \%$ juice intake during the first year had threefold higher odds of obesity. Similarly, inadequate EBF and no SSBs or $100 \%$ juice intake during infancy was associated with a fourfold higher odds of obesity than the referent group. Compared with adequate $\mathrm{BF}$ and no SSBs $100 \%$ juice intake (referent), inadequate BF with SSBs, $100 \%$ fruit juice intake, or both combined were associated with a five- to tenfold higher odds of obesity. Compared with the EBF referent group, inadequate EBF with SSBs, $100 \%$ fruit juice intake, or both combined were associated with a six- to 12 -fold higher odds of obesity. Figure 2 displays the aOR (95\% CIs) of obesity by EBF-beverage groups among all children (aged 25 years) who were exposed to GDM.

\section{Discussion}

This prospective longitudinal analysis showed independent and joint associations for lower BF intensity and shorter duration, and the initiation of SSBs and 100\% fruit juice during the first year of life with subsequent obesity at 2-5 years of age for children exposed to GDM. The odds of child obesity was strongest for the combination of any $\mathrm{BF}$ or $\mathrm{EBF}<6$ months with intake of SSB or $100 \%$ fruit juice during the first year of life: a six- to 12-fold higher odds of obesity compared with $\geq 6$ months of any BF or EBF and no intake of SSBs or $100 \%$ fruit juice. These findings remained after accounting for intrauterine risk factors for child obesity, including maternal hyperglycaemia, pre-pregnancy obesity, gestational weight gain and sociodemographic factors. To our knowledge, this is the first study to evaluate independent and joint associations for BF measures and infant dietary intake of added sugar or naturally sweetened beverages with subsequent child obesity among GDM offspring. Of note, the associations of infant diet of sweetened beverages during the first year of life with child obesity increased markedly for infants with inadequate vs adequate BF (five- to tenfold vs threefold higher odds). The associations persisted after accounting for in utero exposure to maternal obesity, and severity of maternal glucose intolerance among other factors.
Fig. 1 aORs (95\% CIs) for obesity among children aged $2-5$ years exposed to GDM associated with SSB intake and/or $100 \%$ fruit juice during infancy and stratified by $\mathrm{BF}$ duration. $* p<0.05, * * p<0.01, * * * p<0.001$ : significantly higher odds compared with referent;

${ }^{\mathrm{R}}$ Referent: adequate BF, no SSBs, no $100 \%$ fruit juice; adequate $\mathrm{BF}$ : $\mathrm{BF} \geq 6$ months; inadequate $\mathrm{BF}:$ no $\mathrm{BF}$ or $<6$ months BF. Adjusted covariates: child sex, race/ethnicity, parity, WIC participation, maternal education level, prenatal $3 \mathrm{~h}$ OGTT sum of four $z$ scores (GDM severity), GDM treatment type, gestational weight gain, gestational age at GDM diagnosis, and child age at BMI measurement

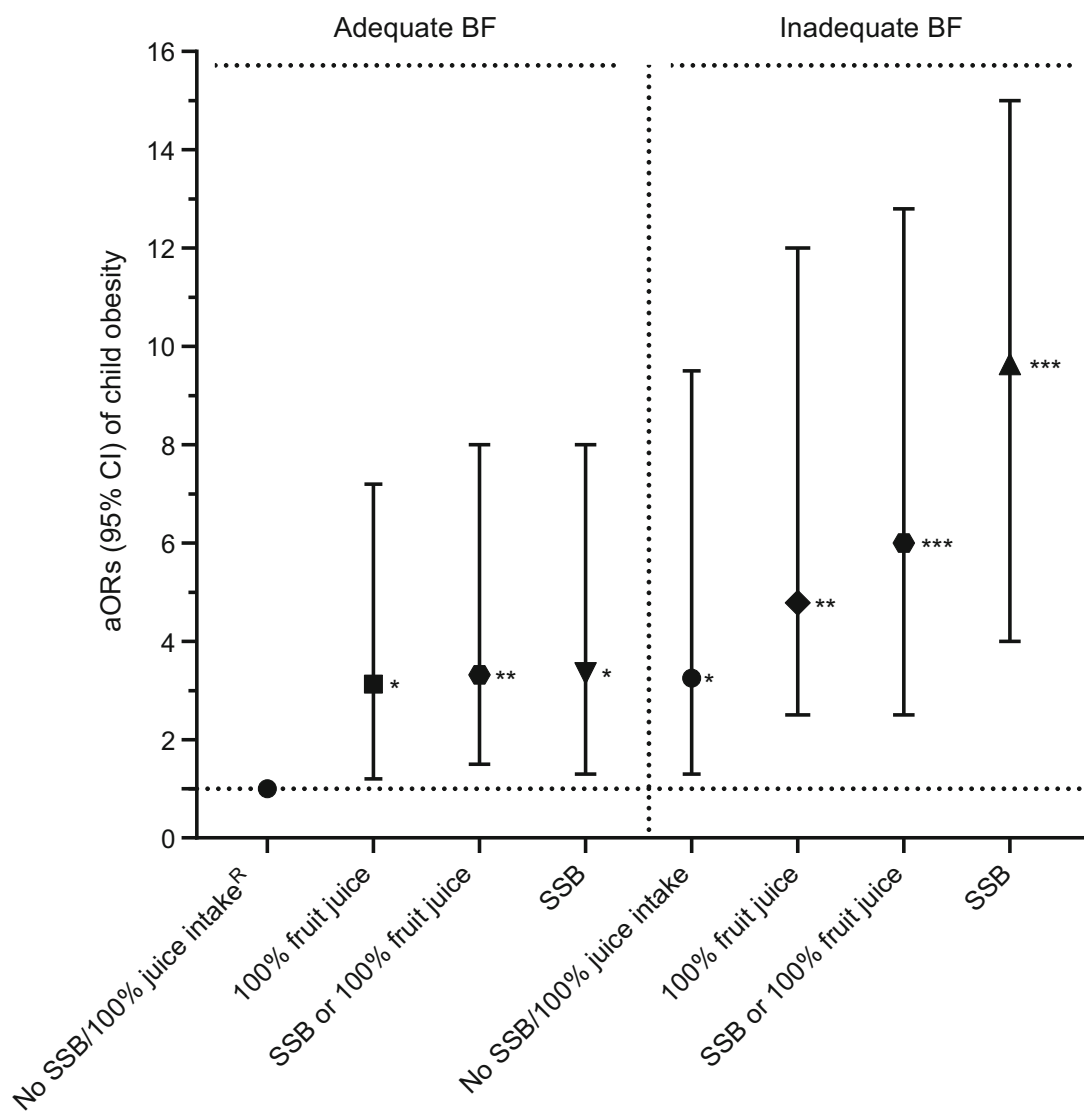


Fig. 2 aORs (95\% CIs) for obesity among children aged 2-5 years exposed to GDM associated with SSB and $100 \%$ fruit juice intake during infancy and stratified by EBF duration. $* * p<0.01, * * * p<0.001$ : significantly higher odds compared with referent;

${ }^{\mathrm{R}}$ Referent: adequate EBF, no SSB, no $100 \%$ fruit juice; adequate EBF: exclusive $\mathrm{BF} \geq 6$ months; inadequate EBF: no EBF or $<6$ months EBF. Adjusted covariates: child sex, race/ethnicity, parity, WIC participation, maternal education level, prenatal $3 \mathrm{~h}$ OGTT sum of four $z$ scores (GDM severity), GDM treatment type, gestational weight gain, gestational age at GDM diagnosis, and child age at BMI measurement

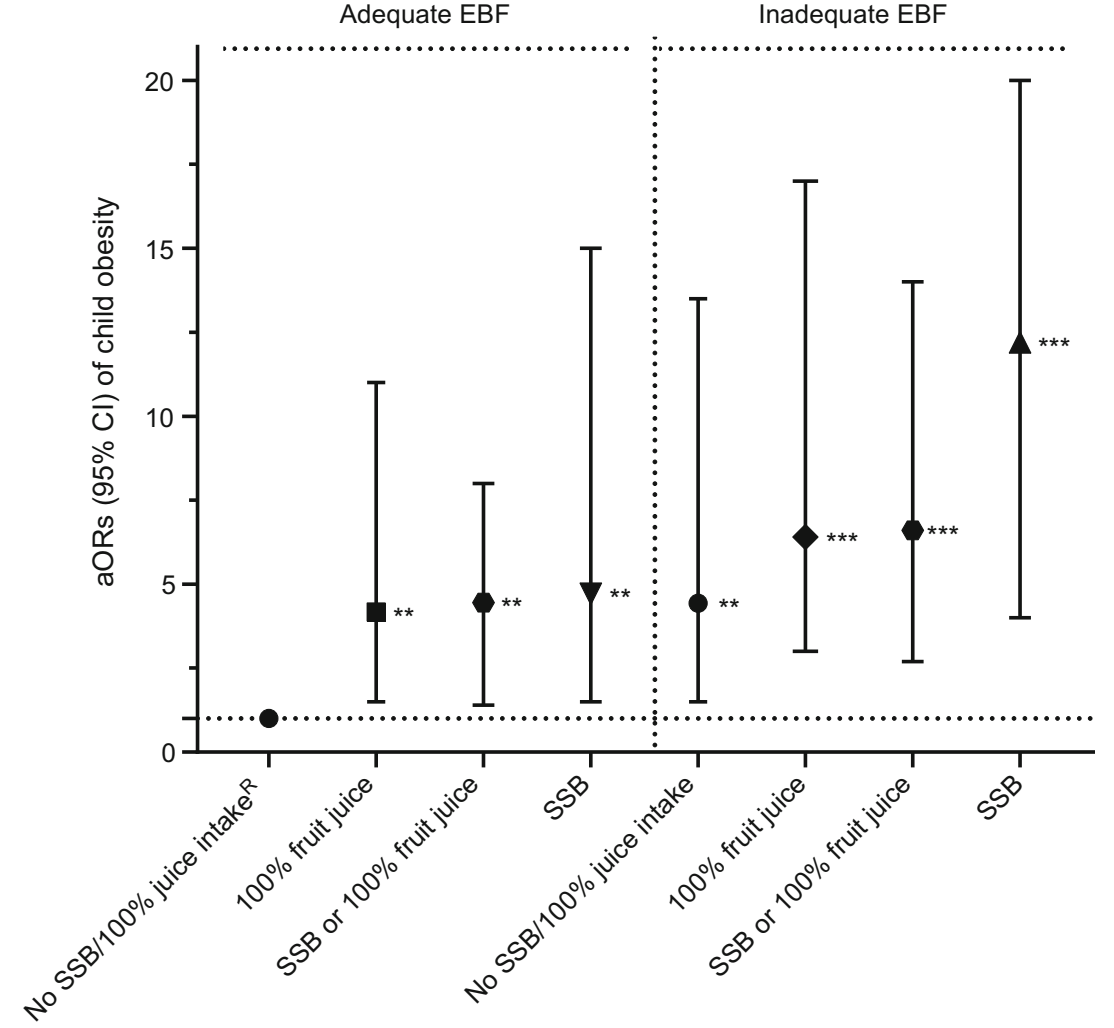

Previous studies, primarily cross-sectional or retrospective designs, have shown inverse associations for EBF duration with BMI-for-age percentile, and/or excessive weight gain [9, $18,19,23]$ or higher prevalence of obesity with concurrent SSBs intake among elementary school-age children or preadolescents exposed to GDM [23]. However, these studies did not assess infant dietary intake, except for maternal recall of BF; in these studies, older children who were exposed to GDM were investigated and the severity of GDM was not assessed. A cross-sectional study of WIC participants found a higher odds of obesity at 1-5 years of age in children who were exclusively breastfed with concurrent SSB intake, regardless of GDM exposure [11]. However, this study did not assess GDM severity or infant diet during the first year of life. The findings are consistent with our previous analysis showing that higher $\mathrm{BF}$ intensity and longer duration were associated with slower longitudinal ponderal growth and lower attained weight-forlength from birth through 1 year of age, independent of prenatal exposure among 464 SWIFT mother-infant dyads enrolled in a substudy of infant growth that involved anthropometric measurements and validated assessment of other infant behaviours in the first year of life [7].

There is mounting evidence that intrauterine exposure to GDM may impact the fetal programming of obesity [34] and increase glucose-linked hypothalamic activation and therefore obesity risk in offspring [35]. Women with GDM are less likely to breastfeed exclusively in the first postpartum hour and are more likely to formula feed their children in the hospital than women without GDM are [36], although adverse clinical perinatal outcomes and maternal obesity may affect the early feeding practices. While evidence for causal associations between $\mathrm{BF}$ and lower child obesity risk in general is substantial, much less is known about the impact of BF intensity and infant dietary habits during the first year of life, specifically, the effect of SSB and fruit juice intake on the early growth and adiposity of young children exposed to GDM $[7,10,11]$. Investigation into human milk constituents such as human milk oligosaccharides, leptin, insulin and other hormones and nutrients in milk of mothers, particularly those with GDM, warrant further study for their putative effects on child obesity rates.

In this prospective study, EBF $\geq 6$ months with added SSBs or $100 \%$ fruit juice increased the odds of obesity at 2-5 years by fourfold compared with no intake of SSBs or $100 \%$ fruit juice, with much stronger associations for any BF combined with beverage intakes. Evidence from prospective studies in the general population is conflicting in relation to $100 \%$ fruit juice intake during infancy and toddlerhood and future child obesity prevalence [37-39]. A meta-analysis of $100 \%$ fruit juice intake in young children has been associated with higher BMI $z$ scores at 1-6 years of age [14], but other studies have shown no effect at older ages (1-18 years) [15]. Another metaanalysis of 4657 articles reported that eight prospective cohort studies with 34,470 children (aged 1-18 years) found direct associations between $100 \%$ fruit juice (177-236 ml/day) consumption and BMI $z$ score controlling for total energy 
intake only for young children aged 1-6 years. Of note, no previous studies have assessed the intake of $100 \%$ fruit juice during the first year of life [14]. In contrast, a systematic review of studies published during 1995-2013 showed no significant associations between concurrent $100 \%$ fruit juice intake and weight or adiposity in children aged 2-5 years [15]. However, intake of SSBs or $100 \%$ fruit juice during the first year of life was not evaluated and findings were not presented for GDM-exposed children. While many studies have shown that SSB intake is directly associated with obesity in children $[8,40,41]$, relatively few have looked at this association prospectively from infancy through to early childhood or pre-adolescence (6 and 8-14 years), and findings are mixed $[42,43]$. Our findings from a large, well-characterised prospective cohort of mother-infant dyads exposed to GDM provide strong evidence that intake of SSBs or naturally sweetened fruit juice at $<12$ months of age markedly increases the risk of obesity in early childhood, and that this risk depends on the intensity and duration of BF.

A considerable amount of calories $(7.6 \%$ of total caloric intake) in the diet of U.S. toddlers comes from SSBs [44]. The mechanisms underlying the independent associations between BF and child obesity are hypothesised to involve satiety responsiveness and lower protein content of breastmilk [45]. How fruit juice and SSB intake during early infancy causes obesity is less clear. Sweet taste and maternal feeding practices related to nutritional habits and soothing children with foods and liquid feeding may be associated with higher daily SSB intake and consequent child obesity [45].

Strengths of this study include the prospective, quantitative research methods used to assess BF measures, and detailed recording of infant dietary intake via monthly mailed surveys, telephone and in-person interviews from birth to 12 months [28]. We also obtained clinical measures of weight before pregnancy or during early gestation to calculate prepregnancy BMI and gestational weight gain. Child height and weight measurements were obtained from KPNC paediatric well-child clinical visits using standardised procedures with calibrated equipment within a single integrated healthcare system. Another major strength is the wellcharacterised research cohort of mother-infant dyads; $95 \%$ of participants were retained at 1-2 years post-baseline, and $83 \%$ of children were still KPNC members at $2-5$ years of age.

This study had some limitations. First, it was not possible to conduct direct observational or physiological measures of BF (i.e. weighing infants) in this large prospective cohort of women with recent GDM. Residual confounding associated with measurement error for beverage and dietary intake covariates is possible; assessing the clinical and sociodemographic characteristics would have reduced this possibility. Later physical activity measurements concurrent with child BMI were unavailable in the EHR. The racially and ethnically diverse SWIFT study of mothers with GDM are representative of women with GDM in the USA. However, Northern California women have higher BF initiation rates than other areas in the USA [46]. The monthly dietary intake assessments determined the age at which SSBs, fruit juice and food items were introduced, but not the specific quantities.

In summary, the combination of shortened BF or EBF and exposure to SSB and/or 100\% fruit juice during the first year of life was associated with six- to 12 -fold higher odds of obesity. This indicates that infant feeding exposures, including sugary beverages, may play a major role in countering fetal life programming, and that modification of early postnatal infant feeding habits may be beneficial in ameliorating the risks to the child from intrauterine exposure to maternal hyperglycaemia.

Our findings add to a growing body of evidence that none or inadequate BF combined with intake of SSBs and $100 \%$ fruit juice during the first year independently and synergistically contribute to future development of child obesity. These findings highlight the need for interventions targeting pregnant women with GDM to focus on promoting higher BF and EBF duration, particularly in mothers with obesity, and to avoid giving infants SSBs and 100\% fruit juice during the first year of life. In conclusion, this study suggests that increasing BF duration and intensity and avoiding SSBs and $100 \%$ fruit juices could combat childhood obesity especially in children exposed to intrauterine GDM.

Supplementary Information The online version of this article (https:// doi.org/10.1007/s00125-020-05379-y) contains peer-reviewed but unedited supplementary material.

Acknowledgements We would like to thank all the SWIFT participants for their contributions to this study. We would like to thank the KPNC, Division of Research SWIFT study team led by EPG for all of their hard work in conducting the in-person research visits and collecting data.

Data availability The KPNC data used for this study is owned by the Kaiser Foundation Health Plan, Kaiser Foundation Hospitals and The Permanente Medical Group; it is not owned by the Kaiser Foundation Research Institute. Because of their third party rights, it is not possible to make the data publicly available without restriction. In accordance with database of record and KPNC's mission to conduct research to serve the public good, we will establish collaborative agreements with other investigators who may wish to use data from this study for future secondary analyses addressing important research questions. Such collaborative relationships will take into account resource constraints, opportunity costs and the need to obtain Institutional Review Board approval.

Funding The analyses were supported by grants from the National Institute of Diabetes, Digestive and Kidney Diseases, R01DK122700 (to EPG), R21 DK103171 (to EPG) and R01DK118409 (to EPG), the National Institute of Child Health and Human Development, R01 HD050625 (to EPG) and the ADA (Clinical Research Award, to EPG).

Authors' relationships and activities The authors declare that there are no relationships or activities that might bias, or be perceived to bias, their work. 
Contribution statement SV, EPG and JND contributed to the overall study design, specific methodologies and acquisition of data, conceptualised the analysis plan, carried out the initial analyses, coordinated the interpretation of results, drafted the initial manuscript and finalised the manuscript. EPG, principal investigator of the SWIFT study, conceived the study design, conducted the data collection, and oversaw the analysis and implementation, and the writing of the manuscript. All authors read and approved the final manuscript. EPG is the guarantor of this work.

\section{References}

1. American Diabetes Association (2019) 2. Classification and diagnosis of diabetes: Standards of medical care in diabetes-2019. Diabetes Care 42:S13-S28. https://doi.org/10.2337/dc19-S002

2. Jiwani A, Marseille E, Lohse N, Damm P, Hod M, Kahn JG (2012) Gestational diabetes mellitus: Results from a survey of country prevalence and practices. J Matern Neonatal Med 25(6):600-610. https://doi.org/10.3109/14767058.2011.587921

3. Dabelea D, Sauder KA (2018) Intrauterine exposure to maternal diabetes and childhood obesity. In: Freemark MS (ed) Pediatric Obesity. Contemporary Endocrinology. Humana Press, Cham, pp 229-242

4. Fryar CD, Carroll MD, Ogden CL (2016) Prevalence of overweight and obesity among children and adolescents aged 2-19 years: United States, 1963-1965 through 2013-2014. NCHS Heal EStats $1-5$

5. Hales CM, Carroll MD, Fryar CD, Ogden CL (2017) Prevalence of obesity among adults and youth: United States, 2015-2016. NCHS Data Brief 288:1-8

6. Faith MS, Hittner JB, Hurston SR et al (2019) Association of infant temperament with subsequent obesity in young children of mothers with gestational diabetes mellitus. JAMA Pediatr 173(5):424- 433. https://doi.org/10.1001/jamapediatrics.2018.5199

7. Gunderson EP, Greenspan LC, Faith MS, Hurston SR, Quesenberry CP (2018) Breastfeeding and growth during infancy among offspring of mothers with gestational diabetes mellitus: a prospective cohort study. Pediatr Obes 13(8):492-504. https://doi. org/10.1111/ijpo.12277

8. Luger M, Lafontan M, Bes-Rastrollo M, Winzer E, Yumuk V, Farpour-Lambert N (2018) Sugar-sweetened beverages and weight gain in children and adults: A systematic review from 2013 to 2015 and a comparison with previous studies. Obes Facts 10(6):674693. https://doi.org/10.1159/000484566

9. Davis JN, Koleilat M, Shearrer GE, Whaley SE (2014) Association of infant feeding and dietary intake on obesity prevalence in lowincome toddlers. Obesity 22(4):1103-1111. https://doi.org/10. 1002/oby.20644

10. Vandyousefi S, Goran MI, Gunderson EP et al (2019) Association of breastfeeding and gestational diabetes mellitus with the prevalence of prediabetes and the metabolic syndrome in offspring of Hispanic mothers. Pediatr Obes 14(7):1-10. https://doi.org/10. 1111/ijpo. 12515

11. Vandyousefi S, Whaley SE, Widen EM et al (2019) Association of breastfeeding and early exposure to sugar-sweetened beverages with obesity prevalence in offspring born to mothers with and without gestational diabetes mellitus. Pediatr Obes 14(12):1070. https:// doi.org/10.1111/ijpo.12569

12. de Seymour J, Chia A, Colega M et al (2016) Maternal dietary patterns and gestational diabetes mellitus in a multi-ethnic Asian cohort: The GUSTO study. Nutrients 8(9):574. https://doi.org/10. 3390/nu8090574

13. Park S, Pan L, Sherry B, Li R (2014) The association of sugarsweetened beverage intake during infancy with sugar-sweetened beverage intake at 6 years of age. Pediatrics 134:S56-S62. https:// doi.org/10.1542/peds.2014-0646J

14. Auerbach BJ, Wolf FM, Hikida A et al (2017) Fruit juice and change in BMI: A meta-analysis. Pediatrics 139(4):e20162454. https://doi.org/10.1542/peds.2016-2454

15. Crowe-White K, O'Neil CE, Parrott JS et al (2016) Impact of 100\% fruit juice consumption on diet and weight status of children: An evidence-based review. Crit Rev Food Sci Nutr 56(5):871-884. https://doi.org/10.1080/10408398.2015.1061475

16. American Pediatric Academy (2020) Infant Food and Feeding. https://www.aap.org/en-us/advocacy-and-policy/aap-healthinitiatives/HALF-Implementation-Guide/Age-Specific-Content/ Pages/Infant-Food-and-Feeding.aspx. Accessed 27 Nov 2020

17. Kleinman RE (2000) American academy of pediatrics recommendations for complementary feeding. Pediatrics 106(5 II):1274

18. Shearrer GE, Whaley SE, Miller SJ, House BT, Held T, Davis JN (2015) Association of gestational diabetes and breastfeeding on obesity prevalence in predominately Hispanic low-income youth. Pediatr Obes 10(3):165-171. https://doi.org/10.1111/ijpo.247

19. Bider-Canfield Z, Martinez MP, Wang X et al (2017) Maternal obesity, gestational diabetes, breastfeeding and childhood overweight at age 2 years. Pediatr Obes 12(2):171-178. https://doi. org/10.1111/ijpo.12125

20. Crume TL, Ogden L, Maligie M et al (2011) Long-term impact of neonatal breastfeeding on childhood adiposity and fat distribution among children exposed to diabetes in utero. Diabetes Care 34(3): 641-645. https://doi.org/10.2337/dc10-1716

21. Kawasaki M, Arata N, Ogawa Y (2018) Obesity and abnormal glucose tolerance in the offspring of mothers with diabetes. Curr Opin Obstet Gynecol 30(6):361-368. https://doi.org/10.1097/ GCO.0000000000000479

22. Taveras EM, Gillman MW, Kleinman KP, Rich-Edwards JW, Rifas-Shiman SL (2013) Reducing racial/ethnic disparities in childhood obesity the role of early life risk factors. JAMA Pediatr 167(8):731-738. https://doi.org/10.1001/jamapediatrics.2013.85

23. Sauder KA, Bekelman TA, Harrall KK, Glueck DH, Dabelea D (2019) Gestational diabetes exposure and adiposity outcomes in childhood and adolescence: An analysis of effect modification by breastfeeding, diet quality, and physical activity in the $\mathrm{EPOCH}$ study. Pediatr Obes 14(12):1-9. https://doi.org/10.1111/ijpo.12562

24. Lim S, Zoellner JM, Lee JM et al (2009) Obesity and sugarsweetened beverages in african-american preschool children: A Longitudinal study. Obesity 17(6):1262-1268. https://doi.org/10. 1038/oby.2008.656

25. Gunderson EP, Matias SL, Hurston SR et al (2011) Study of Women, Infant Feeding, and Type 2 Diabetes mellitus after GDM pregnancy (SWIFT), a prospective cohort study: Methodology and design. BMC Public Health 11:1-15. https://doi.org/10.1186/14712458-11-952

26. Carpenter MW, Coustan DR (1982) Criteria for screening tests for gestational diabetes. Am J Obstet Gynecol 144(7):768-773. https:// doi.org/10.1016/0002-9378(82)90349-0

27. Gunderson EP, Hurston SR, Dewey KG et al (2015) The study of women, infant feeding and type 2 diabetes after GDM pregnancy and growth of their offspring (SWIFT Offspring study): Prospective design, methodology and baseline characteristics. BMC Pregnancy Childbirth 15(1):1-15. https://doi.org/10.1186/s12884-015-0587-Z

28. Piper S, Parks PL (2001) Use of an intensity ratio to describe breastfeeding exclusivity in a national sample. J Hum Lact 17(3): 227-232. https://doi.org/10.1177/089033440101700306

29. Kaiser Permanente (2019) Children's Growth Chart. https:// healthy.kaiserpermanente.org/health-wellness/health- 
encyclopedia/he.children\%27s-growth-chart.hw250189. Accessed 30 Jun 2020

30. WHO Multicentre Growth Reference Study Group (2006) WHO child growth standards based on length/height, weight and age. Acta Paediatr Int J Paediatr 95(SUPPL. 450):76-85. https://doi. org/10.1080/08035320500495548

31. WHO Multicentre Growth Reference Study Group. (1999) The WHO Multicentre Growth Reference Study (MGRS). https:// www.who.int/childgrowth/mgrs/en/. Accessed 30 Jun 2020

32. World Health Organization (2016) The WHO Child Growth Standards. http://www.who.int/childgrowth/standards/en/. Accessed 30 Jun 2020

33. Center for Disease Control and Prevention (2010) Morbidity and mortality weekly report use of World Health Organization and CDC growth charts for children aged $0-59$ months in the United States Department of Health and Human Services. http://www.cdc. gov/mmwr/preview/mmwrhtml/rr5909a1.htm

34. Di Bernardo S, Mivelaz Y, Epure AM et al (2017) Assessing the consequences of gestational diabetes mellitus on offspring's cardiovascular health: MySweetHeart Cohort study protocol, Switzerland. BMJ Open 7(11):e016972. https://doi.org/10.1136/ bmjopen-2017-016972

35. Page KA, Luo S, Wang X et al (2019) Children exposed to maternal obesity or gestational diabetes mellitus during early fetal development have hypothalamic alterations that predict future weight gain. Diabetes Care 42(8):1473-1480. https://doi.org/10.2337/dc182581

36. Oza-Frank R, Chertok I, Bartley A (2015) Differences in breastfeeding initiation and continuation by maternal diabetes status. Public Health Nutr 18(4):727-735. https://doi.org/10.1017/ S1368980014000792

37. Sonneville KR, Long MW, Rifas-Shiman SL, Kleinman K, Gillman MW, Taveras EM (2015) Juice and water intake in infancy and later beverage intake and adiposity: Could juice be a gateway drink? Obesity 23(1):170-176. https://doi.org/10.1002/oby.20927

38. Newby PK, Peterson KE, Berkey CS, Leppert J, Willett WC, Colditz GA (2004) Beverage consumption is not associated with changes in weight and body mass index among low-income preschool children in North Dakota. J Am Diet Assoc 104(7): 1086-1094. https://doi.org/10.1016/j.jada.2004.04.020

39. Faith MS, Dennison BA, Edmunds LS, Stratton HH (2006) Fruit juice intake predicts increased adiposity gain in children from lowincome families: Weight status-by-environment interaction. Pediatrics 118(5):2066-2075. https://doi.org/10.1542/peds.20061117

40. Olsen NJ, Heitmann BL (2009) Intake of calorically sweetened beverages and obesity. Obes Rev 10(1):68-75. https://doi.org/10. 1111/j.1467-789X.2008.00523.x

41. Keller A, Bucher Della Torre S (2015) Sugar-sweetened beverages and obesity among children and adolescents: A review of systematic literature reviews. Child Obes 11(4):338-346. https://doi.org/ 10.1089/chi.2014.0117

42. Cantoral A, Téllez-Rojo MM, Ettinger AS, Hu H, Hernández-Ávila M, Peterson K (2016) Early introduction and cumulative consumption of sugar-sweetened beverages during the pre-school period and risk of obesity at 8-14 years of age. Pediatr Obes 11(1):68-74. https://doi.org/10.1111/ijpo.12023

43. Pan L, Li R, Park S, Galuska DA, Sherry B, Freedman DS (2014) A longitudinal analysis of sugar-sweetened beverage intake in infancy and obesity at 6 years. Pediatrics 134:S29-S35. https://doi.org/10. 1542/peds.2014-0646F

44. Rosinger A, Herrick K, Gahche J, Park S (2017) Sugar-sweetened beverage consumption among U.S. youth, 2011-2014. NCHS Data Brief 271:1-8

45. Bartok CJ, Ventura AK (2009) Mechanisms underlying the association between breastfeeding and obesity. Int J Pediatr Obes 4(4): 196-204. https://doi.org/10.3109/17477160902763309

46. Center for Disease Control and Prevention (2018) Breastfeeding Report Card | Breastfeeding. https://www.cdc.gov/breastfeeding/ data/reportcard.htm. Accessed 30 Jun 2020

Publisher's note Springer Nature remains neutral with regard to jurisdictional claims in published maps and institutional affiliations. 